\title{
Deformation of a Liquid Film by an Impinging Gas Jet: Modelling and Experiments
}

\author{
Chinasa J. Ojiako ${ }^{1}$, Radu Cimpeanu ${ }^{2}$, Hemaka Bandulasena ${ }^{3}$, Roger Smith $^{1}$, Dmitri Tseluiko $^{1}$ \\ ${ }^{1}$ Department of Mathematical Sciences, Loughborough University \\ LE11 3TU, Loughborough, United Kingdom \\ C.J.Ojiako@lboro.ac.uk; R.Smith@lboro.ac.uk; D.Tseluiko@lboro.ac.uk \\ ${ }^{2}$ Mathematical Institute, University of Oxford \\ OX2 6GG, Oxford, United Kingdom \\ Radu.Cimpeanu@maths.ox.ac.uk \\ ${ }^{3}$ Department of Chemical Engineering, Loughborough University \\ LE11 3TU, Loughborough, United Kingdom \\ H.C.H.Bandulasena@lboro.ac.uk
}

\begin{abstract}
We consider liquid in a cylindrical beaker and study the deformation of its surface under the influence of an impinging gas jet. Analyzing such a system not only is of fundamental theoretical interest, but also of industrial importance, e.g., in metallurgical applications. The solution of the full set of governing equations is computationally expensive. Therefore, to obtain initial insight into relevant regimes and timescales of the system, we first derive a reduced-order model (a thin-film equation) based on the long-wave assumption and on appropriate decoupling the gas problem from that for the liquid and taking into account a disjoining pressure. We also perform direct numerical simulations (DNS) of the full governing equations using two different approaches, the Computational Fluid Dynamics (CFD) package in COMSOL and the volume-of-fluid Gerris package. The DNS are used to validate the results for the thinfilm equation and also to investigate the regimes that are beyond the range of validity of this equation. We additionally compare the computational results with experiments and find good agreement.
\end{abstract}

Keywords: Thin-film equation, DNS, Film rupture, Dewetting, Gas-liquid interface.

\section{Introduction}

The study of a gas jet impinging on a liquid surface is of great interest as it finds application in industries, such as the steel and chemical industries. The liquid surface deforms under the influence of an impinging gas jet and a cavity forms in the center. The interaction of a gas jet with a liquid surface has been investigated by several authors and various factors influencing the deformation of the liquid surface have been analyzed, e.g. the effects of the gas jet velocity [1], the diameter of the gas jet nozzle, the distance from the nozzle to the undisturbed liquid surface [2] and the liquid properties [3]. In previous studies it was found out that the cavity may be stable or unstable, and different unstable regimes were identified, an oscillating cavity and splashing (e.g. [4]), but such regimes have not been analyzed in detail.

In the previous analytical and computational work, the fluid flow in the liquid was either neglected or was not analyzed in detail. We present a study of the dynamics of eddies in the liquid that are generated under the influence of an impinging gas jet on a liquid surface. Such eddies result from the tangential drag of the gas on the liquid. This is of great importance for applications as eddies in the liquid region affect mixing and mass transfer. Since, the solution of the full set of governing equations is computationally expensive, we first develop a reduced-order model (a thin film equation) based on the longwave assumption and on decoupling the gas problem from that for the liquid. This is possible under suitable conditions, namely, when the liquid layer is thin and the typical liquid velocity is sufficiently smaller than that of the gas [5]. The thinfilm equation provides initial insight into the relevant flow regimes and timescales. To analyze a possible rupture of a liquid film and its dewetting, we also introduce the disjoining pressure into our model. We also perform DNS of the full governing equations using two different approaches, the CFD package in COMSOL and the volume-of-fluid Gerris package. The DNS are used to validate the results for the thin-film equation and also to investigate the regimes that are beyond the range of validity of this equation (e.g., when the liquid layer is thicker). The computational results are compared with experiments. 
The rest of the paper is organized as follows: In Sect. 2, we state the problem, introduce the experimental setup and discuss the governing equations. In Sect. 3, we introduce the thin-film equation. The results are discussed in Sect. 4, and the conclusions are given in Sect. 5.

\section{Problem statement and governing equations}

We consider liquid in a cylindrical beaker and study the deformation of its surface under the influence of an impinging gas jet that is generated by maintaining a constant gas flow rate from a stationary cylindrical tube (nozzle) with its axis coinciding with the axis of the beaker, as show in Fig. 1(a). We denote the radius of the beaker by $R_{b}$ and its height by $H$. The thickness of the undisturbed liquid layer is denoted by $h_{0}$. The inner and outer radii of the nozzle are denoted by $R_{i}$ and $R_{0}$, respectively, and the distance between the nozzle and the undisturbed gas-liquid interface is denoted by $h_{1}$. We introduce cylindrical polar coordinates $(R, \varphi, z)$ with the $z$-axis pointing upwards along the axis of the beaker in the direction opposite to gravity $g$, and with the bottom of the beaker coinciding with the $z=0$ plane. The deformed gas-liquid interface is located at $z=h(R, \varphi, t)$. For simplicity, we will assume that the liquid and the gas are of the same constant temperature.

(a)

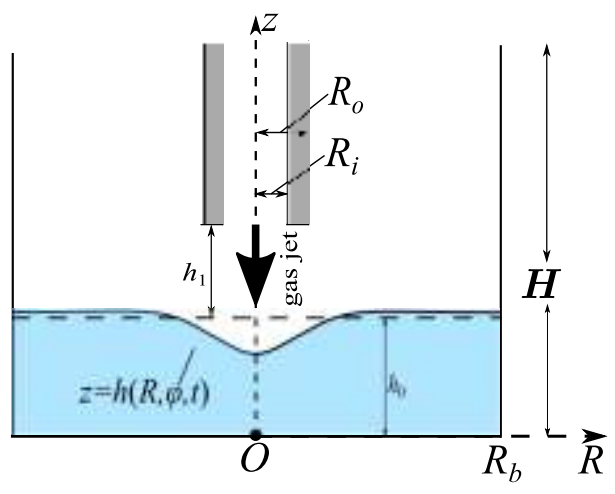

(b)

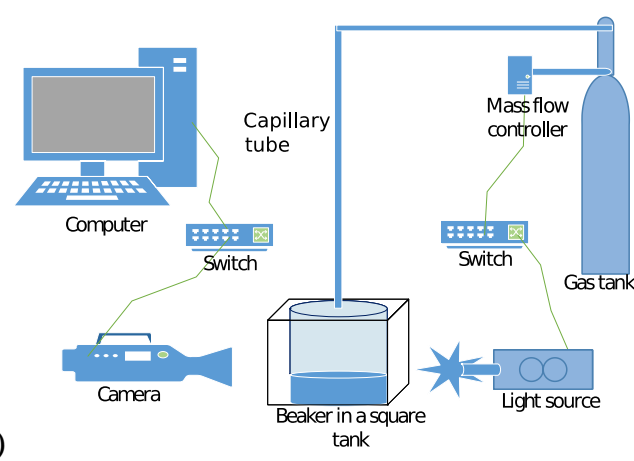

Fig. 1: (a) Schematic representation of a gas jet impinging on a liquid surface in a cylindrical beaker. (b) Experimental set up.

Figure 1(b) shows the experimental set-up of the system. The gas from a gas tank flows through a cylindrical pipe and is supplied via a mass flow controller (MKS, PR4000B) which regulates the gas flow rate. As the gas jet strikes the liquid surface at the center, the liquid surface deforms which causes a cavity in the liquid. The interface evolution was measured using a high-speed camera (Photon Fastcam, M2.1) coupled with long distance lens (Infinity, KC) which is connected to a computer to store data. For the high-speed video recording, we provide illumination using a light source (Kern Dual Fiber Unit LED). The recording was done at 2000 frames per second (fps) and saved at $30 \mathrm{fps}$ for analysis. These experiments were repeated 8 times to collect sufficient number of images for analysis.

As the typical velocity in the liquid will be small compared to the speed of sound in the liquid, it is appropriate to model the flow in the liquid by the incompressible Navier-Stokes equations. However, as the gas flow can be relatively fast (e.g. up to $100 \mathrm{~m} / \mathrm{s}$ ), the flow in the gas will be modelled by the compressible Navier-Stokes equations (with an additional equation of state - we use the ideal gas relation).

At the nozzle boundary and at the bottom of the beaker, we impose no-slip and no-penetration conditions. At the side wall of the beaker, we again assume no-penetration both for the liquid and for the gas. However, to resolve the stress singularity at the contact line, instead of imposing the no-slip condition, we impose the Navier slip condition both for the liquid and for the gas [6]. We also impose a fix contact angle at the contact line. At the gas inlet, we impose a fixed parabolic velocity profile, corresponding to the fully developed Poiseuille flow in the nozzle. At the gas outlet, we impose normal flow and prescribe normal stress. Finally, we have the dynamic balance of stress at the gas-liquid interface:

$$
\boldsymbol{n} \cdot \boldsymbol{\sigma}_{\boldsymbol{l}}+(-\boldsymbol{n}) \cdot \boldsymbol{\sigma}_{\boldsymbol{g}}=\gamma \kappa \boldsymbol{n} .
$$


Here, $\boldsymbol{n}$ is the unit normal vector to the interface pointing into the liquid. $\boldsymbol{\sigma}_{l}=-p_{l} \boldsymbol{\delta}+\mu_{l}\left(\nabla \boldsymbol{u}^{T}+\nabla \boldsymbol{u}\right)$ is the stress tensor in the liquid with $\boldsymbol{\delta}$ denoting the identity tensor, $\mu_{l}$ denoting the dynamic viscosity of the liquid and $p_{l}$ and $\boldsymbol{u}$ denoting the pressure and the velocity in the liquid. $\boldsymbol{\sigma}_{g}=-p_{g} \boldsymbol{\delta}+\mu_{g}\left(\nabla \boldsymbol{v}^{T}+\nabla \boldsymbol{v}\right)-\frac{2}{3} \mu_{g}(\nabla \cdot \boldsymbol{v}) \boldsymbol{\delta}$ is the stress tensor in the gas with $\mu_{g}$ denoting the dynamic viscosity of the gas and $p_{g}$ and $\boldsymbol{v}$ denoting the pressure and the velocity in the gas. Also, $\gamma$ is the gasliquid surface tension coefficient (which is assumed to be constant) and $\kappa=\nabla \cdot \boldsymbol{n}$ is the curvature of the interface.

\section{Thin-film equation}

Solving the full system of equations governing the problem of a gas jet impinging on a liquid surface is a computationally expensive task, and the aim here is to derive a reduced-order model which still captures the important physics of the problem. The first step is to decouple the problem for the gas from that for the liquid, which is appropriate when the typical velocity in the liquid is much smaller than that in the gas. Then, for the gas problem it is appropriate to neglect the motion of the liquid and to use the quasi-static assumption $[5,7,8]$. The solution for the gas problem can then be used to obtain the stress exerted by the gas onto the gas-liquid interface, $\boldsymbol{s}_{g}=-\boldsymbol{n} \cdot \boldsymbol{\sigma}_{g}$. For the liquid, we use the thin-film approximation, assuming that the film thickness $h_{0}$ is small compared to the horizontal length scale $l$, and we introduce the so called thin-film parameter $\varepsilon=$ $h_{0} / l$. Assuming axial symmetry (i.e., no dependence on $\varphi$ ), and using a systematic asymptotic expansion in the thin-film parameter we obtain the following thin-film equation at leading order

$$
h_{t}+\frac{1}{\mu_{l}} \frac{1}{R}\left(-\frac{R h^{3}}{3} p_{l R}+\frac{R h^{2}}{2} T_{s}\right)_{R}=0 .
$$

Here, the subscripts $t$ and $R$ denote partial derivatives with respect to these variables, $p_{l}=-\rho_{l} g(z-h)+N_{s}-$ $\gamma\left(R h_{R}\right)_{R} / R-\Pi(h)$ is the leading-order pressure in the liquid with $\rho_{l}$ denoting the density of the liquid and $g$ denoting gravity. $\Pi(h)=-A / h^{3}+B / h^{6}$ is the disjoining pressure accounting for the wettability of the liquid, with $A$ and $B$ being constants determining short-range and long-range interactions [9]. The contact angle and the precursor film thickness are determined by $A$ and $B$ (see [9]). Also, $N_{s}$ and $T_{s}$ are the normal and tangential (in the radial direction) components of $\boldsymbol{s}_{g}$.

\section{Results}

First, we consider water of thickness of $0.05 \mathrm{~cm}$ in a beaker of height $3.5 \mathrm{~cm}$ and diameter $3 \mathrm{~cm}$. An air jet of 0.2 standard liters per minute (slpm) flows through a nozzle diameter of $0.16 \mathrm{~cm}$ and is fixed at the distance $0.5 \mathrm{~cm}$ away from the undisturbed gas-liquid interface. For the thin-film model, we first solve the gas-only problem by replacing the gas-liquid interface with a flat solid wall to obtain the normal and tangential stresses exerted by the gas, $N_{S}$ and $T_{S}$. The resulting gas flow pattern is shown in Fig. 2(a) and the resulting normal and tangential stresses are shown in Fig. 2(b). Using these stresses, we solve the thin-film equation in Matlab, and the resulting steady-state gas-liquid interface and a recirculation region in the liquid are shown in Fig. 2(c). For the gas flow rate of $1 \mathrm{slpm}$, the water ruptures in the center and dewets until reaching a steady state, see Fig. 3(a) for the evolution of the solution of the thin-film equation and Fig. 3(b) for the COMSOL steadystate solution. We also analyze the flow patterns in the liquid as the liquid thickness increases. When the water film is sufficiently thin, only one eddy is generated in the film, see Fig. 2(c). However, the number of eddies increases as the water thickness increases, see Fig. 4(a) showing the flow pattern in the water of thickness $0.5 \mathrm{~cm}$ when the gas flow rate is $1 \mathrm{slpm}$. Figure 4(b) additionally compares the interface shapes obtained using the thin-film model, COMSOL, Gerris and experiment. The DNS results are in very good agreement with the experimental data and the thin-film equation also agrees well with the experiment (even though the water thickness is relatively thick). Figure 4(c) shows the evolution of the minimum point of the interface over time (in COMSOL), and we can observe that the steady state is reached via decaying oscillations. The accuracy of the results has been systematically tested via mesh refinements. 
(a)

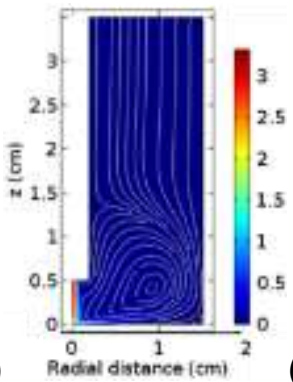

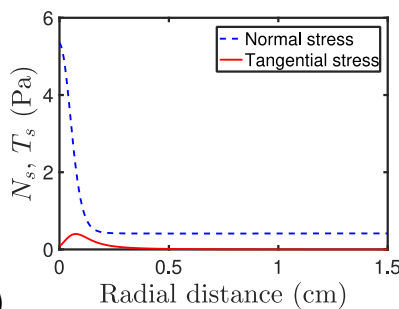

(b)

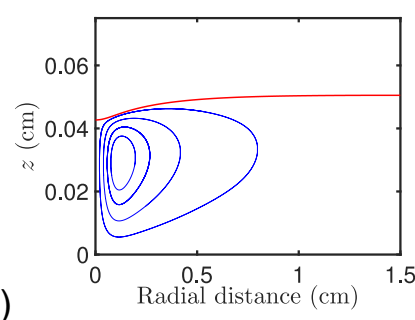

(c)

Fig. 2: (a) An air jet striking the lower wall of a beaker (the color bar correspond to the velocity magnitude). (b) Pressure and shear stress exerted by the air jet on the lower wall of the beaker. (c) The steady-state interface at $t=0.25 \mathrm{~s}$ obtain by solving (2).

(a)

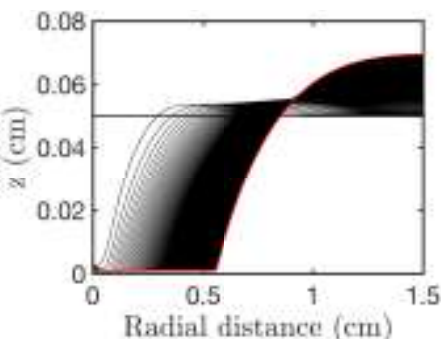

(b)

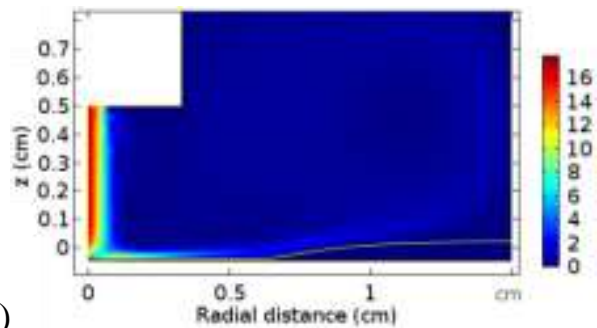

Fig. 3: (a) Evolution of the water surface and dry spot obtained using (2). The black lines show the interfaces at equal time intervals $\Delta t=6.6 \times 10^{-4} \mathrm{~s}$. The red line is the steady-state interface at $t=0.2 \mathrm{~s}$. (b) The steady-state interface obtained using COMSOL.

(a)

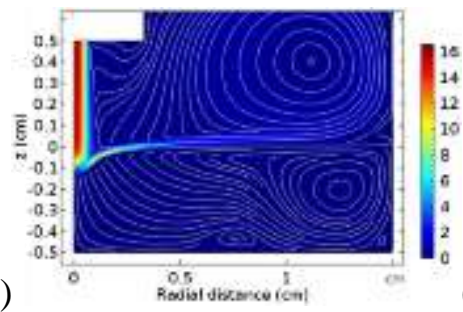

(b)

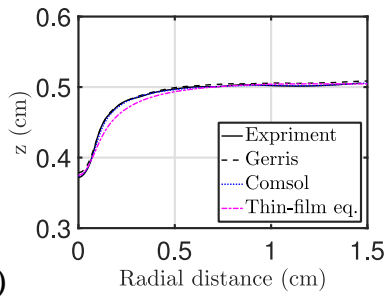

(c)

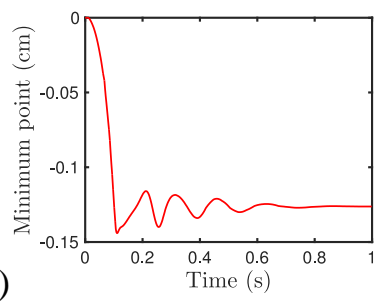

Fig. 4: Water thickness: $0.5 \mathrm{~cm}$. Gas flow rate: $1 \mathrm{slpm}$. (a) Flow pattern when a steady state is reached in COMSOL. (b) Comparison of the experimental interface shape with DNS and the thin-film equation. (c) The minimum point of the cavity plotted against time.

\section{Conclusion}

We analyzed the deformation of a liquid surface and the resulting flow patterns under the action of a gas jet. We used both a reduced-order model and DNS for the full equations and compared the numerical results with experiments. For a thin film and at a relatively low gas flow rate, flow in the liquid evolves into a steady state with one recirculation region and with the interface having a cavity in the centre. At a larger gas flow rate, the liquid film ruptures in the center and dewets until reaching a steady state. For a thicker liquid layer, the flow in the liquid again evolves into a steady state at sufficiently small gas velocities. However, the number of the recirculation regions in the liquid increases as the liquid thickness increases. The study of flow patterns in the liquid is important for applications as it affects e.g. mass transfer.

\section{References}

[1] R. B. Banks and D. V. Chandrasekhara, "Experimental investigation of the penetration of a high-velocity gas jet through a liquid surface," J. Fluid Mech., vol. 15, pp.13-34, 1963.

[2] E. T. Turkdogan, "Fluid dynamics of gas jets impinging on surface of liquids," Chem. Eng. Sci., vol. 21, pp. 1133-1144, 1966. 
[3] N. A. Molloy, "Impinging jet flow in a 2-phase system-basic flow pattern," J. Iron Steel Inst., vol. 208, pp. 943-950, 1970.

[4] R. S. Rosler and G. H. Stewart, "Impingement of gas jets on liquid surfaces," J. Fluid Mech., vol. 31, pp. 163-174, 1968.

[5] E. O. Tuck, "On air flow over free surfaces of stationary water," J. Austral. Math. Soc. (Ser. B), vol. 19, pp. 66-80, 1975.

[6] D. N. Sibley, N. Savva and S. Kalliadasis, "Slip or not slip? A methodical examination of the interface formation model using two-dimensional droplet spreading on a horizontal planar substrate as a prototype system," Phys. Fluids, vol. 24, pp. 082105, 2012.

[7] D. Tseluiko and S. Kalliadasis, "Nonlinear waves in counter-current gas-liquid film flow," J. Fluid Mech., vol. 673, pp. 19-59, 2011.

[8] R. Vellingiri, D. Tseluiko, N. Savva and S. Kalliadasis, "Dynamics of a liquid film sheared by a co-flowing turbulent gas," Int. J. Multiphase Flow, vol. 56, pp. 93-104, 2013.

[9] M. Galvagno, D. Tseluiko, H. Lopez and U. Thiele, "Continuous and discontinuous dynamic unbinding transitions in drawn film flow," Phys. Rev. Lett., vol. 112, pp. 137803, 2014. 